\title{
Topology Optimization of Mechanical Structures Targeting Vibration Characteristics"
}

\author{
Shinji NISHIWAKI**, Yasunori MAEDA ${ }^{* *}$, Kazuhiro IZUI**, \\ Masataka YOSHIMURA $^{* *}$, Kazumi MATSUI ${ }^{* * *}$ and Kenjiro TERADA ${ }^{* * * *}$ \\ **Kyoto University, \\ Yoshida Honmachi, Sakyo-ku, Kyoto, Japan \\ E-mail: shinji@prec.kyoto-u.ac.jp \\ ${ }^{* * *}$ Yokohama National University, \\ Tokiwadai Hodogaya, Yokohama, Japan \\ ****Tohoku University, \\ Aramaki-Aza 06 Aoba, Sendai, Japan
}

\begin{abstract}
This paper proposes a structural topology optimization method for obtaining mechanical structures incorporating desired vibration characteristics for the design of mechanical resonators and vibromotors. First, a new type of homogenization design method is introduced, in which continuous material distributions are assumed using a continuous interpolation function at each node, in order to overcome numerical instabilities such as checkerboard patterns. Next, three design requirements for implementing specific vibration characteristics in the mechanical structures are clarified, namely eigen-frequency matching, eigen-mode control, and stiffness, and the corresponding objective functions are formulated. A new type of multi-objective function incorporating these three objective functions is proposed. The optimization problem is formulated using this multi-objective function, and an optimization algorithm is developed using Sequential Linear Programming (SLP). Finally, several examples are provided in order to confirm the usefulness of the generated optimal designs for vibrating mechanical structures.
\end{abstract}

Key words: Optimum Design, Structural Analysis, Vibration of Continuous System, Sensitivity Analysis, Finite Element Method

\section{Introduction}

Resonance phenomena often lead to instabilities in mechanical structures, and must be avoided for mechanical designs. The maximization of eigen-frequencies ${ }^{(1)-(4)}$ is formulated as the objective function to avoid such resonance phenomena in optimum designs. However, if resonance phenomena can be appropriately controlled and utilized, new dynamic functions or performance capabilities can be implemented in a mechanical structure. Typical examples are found in mechanical resonators and vibromotors, and the former are usually used as dynamic sensors such as acceleration and yaw rate sensors ${ }^{(5),(6)}$. Mechanical resonators are also used as band-pass filters ${ }^{(7)}$ in MEMS by exploiting high Q-value characteristics. On the other hand, vibromotors are utilized as devices for moving a workpiece in either translational ${ }^{(8)}$ or rotational directions ${ }^{(9)}$ by exploiting specific vibration modes.

However, structural optimization methods for the design of mechanical structures having the above dynamic functions have not been established yet, and such structures are thus currently designed by trial and error methods that depend in large part on designer 
experience and intuition. As a result, a great deal of time is often required to develop successful structures, yet these do not always optimally satisfy the design requirements, or reach truly high performance goals.

Topology optimization methods ${ }^{(10)}$ can overcome the above problems since they can create high performance designs that satisfy the design requirements under specified constraints based on mechanics and mathematics. Nishiwaki et al. ${ }^{(11)}$ and Tcherniak ${ }^{(12)}$ obtained mechanical structures incorporating dynamic functions by maximizing output displacements when periodic loads are applied, based on topology optimization methods. However, limitations were encountered in obtaining optimal configurations having physical meaning, due to the extreme multimodal characteristic of the objective function. Even though those particular methods successfully provided optimal solutions, the obtained optimal configurations only made use of the lowest eigen-mode to maximize the output response. Furthermore, direct control of specified eigen-modes was problematic, so this highly desirable capability for the design of the vibromotors could not be fully exploited.

In order to mitigate these problems, we develop a new topology optimization method that can implement target eigen-frequencies and their corresponding eigen-modes, and deliver higher performance mechanical structures than is possible by the direct maximization of output displacements. That is, as explained in Section 2, we first extend a new topology optimization method, in which continuous material distributions are assumed using a continuous interpolation function at each node, to dynamic problems. In Section 3, the design requirements for the mechanical structures having dynamic functions are clarified, and the objective functions to achieve the design requirements are formulated. Based on these formulations, a topology optimization algorithm is constructed using the homogenization method and Sequential Linear Programming (SLP). In Section 4, simple two-dimensional design examples are presented in order to confirm the proposed method.

2. Relaxation of Design Domain Based on Continuous Approximations of Materials

Consider the design problem of determining the boundary of a design domain $\Omega_{d}$ by minimizing or maximizing objective functions. The key idea of the topology optimization method is the introductions of a fixed, extended design domain $D$ that includes the original design domain $\Omega_{d}$, a priori, and the following characteristic function:

$$
\chi_{\Omega}(\boldsymbol{x})= \begin{cases}1 & \text { if } \boldsymbol{x} \in \Omega_{d} \\ 0 & \text { if } \boldsymbol{x} \in D \backslash \Omega_{d}\end{cases}
$$

where $\boldsymbol{x}$ denotes a position in the extended design domain $D$. Using this function, the original structural design problem is replaced by a material distribution problem, and an arbitrary structural configuration can be expressed using this characteristic function. Unfortunately, this characteristic function is often very discontinuous in the fixed design domain.

In order to overcome this problem and obtain continuous functions in the global sense, a homogenization method is used where microstructures are continuously distributed almost everywhere in the extended design domain $D$. Although this status must hold even after finite element discretization, the design variables representing the microstructure distribution status are approximated by piecewise constants in the finite element implementation $^{(13)}$, and such approximation can cause numerical instabilities such as checkerboard patterns. Several numerical techniques such as filtering schemes ${ }^{(14) \sim(16)}$ have been proposed in order to deal with these numerical instability problems but these methods largely depend on artificial parameters for which there are no rational guidelines for determining appropriate a priori parameters. Here, in order to overcome the root cause of the above problems, first, the design variables $r(\boldsymbol{x})$ are allocated at each node in the finite element discretization, and we then assume that the function representing the design 
variable distributions holds the $C^{0}$-continuity over the fixed design domain $D$, and is assumed to be expressed as,

$$
r \approx r^{h}=\boldsymbol{M}(\boldsymbol{x}) \boldsymbol{R}=N_{1}^{r} R_{1}+\cdots+N_{i}^{r} R_{i}+\cdots+N_{n}^{r} R_{n}
$$

where $h$ stands for a discretized quantity using the FEM, $\boldsymbol{M}(\boldsymbol{x})$ is a vector whose components are $N_{i}^{r}(\boldsymbol{x})(i=1, \ldots, n), \boldsymbol{R}$ a vector of nodal (discrete) design variables $R_{i}(i=1, \ldots, n)$, and $n$ is the total number of design variables. Using the above approximation, the design variables are continuously distributed in and throughout the elements, and can avoid the numerical instabilities encountered in $C^{-1}$-continuity. The bi-linear interpolation function is used as $N_{i}^{r}(\boldsymbol{x})$ in the case of four-node quadrilateral elements for its simplicity in this research.

Figure 1 shows the microstructure used for the relaxation of the design domain in the two-dimensional problem. As shown in this figure, its shape is hexagonal and the design variable is a geometrical parameter $r$. In order to for a unit cell to be void, $r$ must be 1 , and for it to be solid material, $r$ must be 0 , and the following constraint must be satisfied:

$$
0 \leq r(x) \leq 1
$$

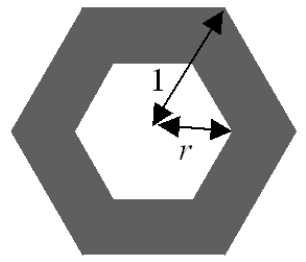

Fig. 1 Hexagonal microstructure

Using this microstructure, the homogenized physical properties are computed in the following procedure. First, the characteristic function $\chi$ is obtained by

$$
\int_{Y} \boldsymbol{\varepsilon}_{y}(\boldsymbol{v})^{T} \boldsymbol{E}(\boldsymbol{x}, \boldsymbol{y}) \boldsymbol{\varepsilon}_{y}(\chi(\boldsymbol{x}, \boldsymbol{y})) d Y=\int_{Y} \boldsymbol{\varepsilon}_{y}(\boldsymbol{v})^{T} \boldsymbol{E}(\boldsymbol{x}, \boldsymbol{y}) d Y \text { for } \forall \boldsymbol{v} \in V_{y}
$$

where $\boldsymbol{y}$ is the local coordinate defined in the microstructure, $\boldsymbol{E}$ is the elasticity tensor of the microstructure, and $V_{y}$ is the admissible space defined in unit cell $Y$ such that

$$
\begin{aligned}
& V_{y}=\left\{\boldsymbol{v}=v_{i} \boldsymbol{e}_{i}: v_{i} \in H^{1}(Y) \text { with } Y \text {-periodic in unit cell } Y\right\} \\
& \boldsymbol{\varepsilon}_{y}(\boldsymbol{v})^{T}=\left\{\frac{\partial v_{1}}{\partial y_{1}} \quad \frac{\partial v_{2}}{\partial y_{2}} \quad \frac{1}{2}\left(\frac{\partial v_{1}}{\partial y_{2}}+\frac{\partial v_{2}}{\partial y_{1}}\right)\right\}
\end{aligned}
$$

Using the value of the characteristic function $\chi$ obtained by the above equation, the homogenized elasticity tensor $\boldsymbol{E}^{H}$ and homogenized mass density $\rho_{m}^{H}$ are calculated as,

$$
\begin{aligned}
& \boldsymbol{E}^{H}=\frac{1}{|Y|} \int_{Y} \boldsymbol{E}(\boldsymbol{x}, \boldsymbol{y})\left[\boldsymbol{I}-\boldsymbol{\varepsilon}_{y}(\chi)\right] d Y \\
& \rho_{m}^{H}=\frac{1}{|Y|} \int_{Y} \rho_{m}(\boldsymbol{x}, \boldsymbol{y}) d Y
\end{aligned}
$$

where $\rho_{m}$ is the mass density of the microstructure, and $|Y|$ stands for the area of the unit cell. We thus obtain the homogenized properties that are then utilized in the optimization procedure.

\section{Formulations}

\subsection{Objective Function Settings}

Let us consider that a two-dimensional design domain $\Omega_{d}$, composed of a linear elastic material, is fixed at boundary $\Gamma_{u}$. Now, the amplitude of periodically oscillating displacement along the direction of a unit load vector $\boldsymbol{t}_{j}^{\text {Outmax }}$ at boundary $\Gamma_{t j}^{\text {Outmax }} \quad(j=1, \ldots$, $\left.m_{s}\right)$ is to be maximized, and the amplitude of periodically oscillating displacement along the direction of a unit load vector $\boldsymbol{t}_{j}^{\text {Outmin }}$ at boundary $\Gamma_{t j}^{\text {Outmin }}$ is to be minimized 
simultaneously when a harmonic exciting traction $\boldsymbol{t}_{j}^{\mathrm{In}}$ is applied at boundary $\Gamma_{t j}^{\mathrm{In}}$, where the $j$-th excitation frequency is assumed to be $\omega_{j}^{\text {In }}$, using $m_{s}$ eigen-frequencies and their corresponding eigen-modes. Here, instead of directly maximizing or minimizing the specified amplitude of vibration of the excitation eigen-frequencies, the desired optimal configurations are obtained by satisfying the following design requirements:

The first design requirement pertains to the eigen-frequency specifications. Suppose that the $j$-th eigen-frequency and its corresponding eigen-mode of the vibrating structure occupying the design domain $\Omega_{d}$ are $\omega_{j}$ and $\phi_{j}$, respectively. One of the eigen-frequencies must match the excitation frequency $\omega_{j}^{\text {In }}$ in order to obtain the desired maximization of the displacement. Here, we intend to match the $j$-th eigen-frequency $\omega_{j}$ with the $j$-th excitation frequency $\omega_{j}^{\text {In }}\left(j=1, \ldots, m_{s}\right)$. Doing so, however, raises the possibility the changes may occur in the sequence in which the eigen-frequencies and eigen-modes are dealt with during the optimization process. In order to avoid this reordering, an appropriate number, $m-m_{s}\left(m>m_{s}\right)$, of fictitious target eigen-frequencies $\omega_{m_{s}+1}^{\text {In }}, \omega_{m_{s}+2}^{\text {In }}, \ldots, \quad \omega_{m}^{\text {In }}$ must be given sufficiently large values, and the matching of eigen-frequencies with target values is also taken into account. Thus, the objective function is formulated as,

$$
\underset{r}{\operatorname{minimize}} f_{j}^{V}=\left|\frac{\omega_{j}(r)^{2}-\omega_{j}^{\operatorname{In} 2}}{\omega_{j}^{\operatorname{In} 2}}\right| \text { for } j=1, \ldots, m
$$

The second design requirement pertains to the eigen-mode specifications. The maximization and minimization of the amplitudes of the displacements at specified boundaries are achieved by the eigen-mode specification, formulated as,

$$
\begin{aligned}
& \underset{r}{\operatorname{maximize}} f_{j}^{M, \operatorname{In}}=\left|\int_{\Gamma_{t j}^{\ln }} \boldsymbol{t}_{j}^{\operatorname{In} T} \phi_{j}(r) d \Gamma\right|, \\
& \underset{r}{\operatorname{maximize}} f_{j}^{M, \text { Outmax }}=\left|\int_{\Gamma_{i j}^{\text {Outmax }}} \boldsymbol{t}_{j}^{\text {Outmax } T} \boldsymbol{\phi}_{j}(r) d \Gamma\right| \text {, } \\
& \text { and } \underset{r}{\operatorname{minimize}} \quad f_{j}^{M, \text { Outmin }}=\left|\int_{\Gamma_{t j}^{\text {Outmin }}} \boldsymbol{t}_{j}^{\text {Outmin } T} \boldsymbol{\phi}_{j}(r) d \Gamma\right| \quad \text { for } j=1, \ldots, m_{s}
\end{aligned}
$$

Here, the $j$-th eigen-mode is $\phi_{j}$ obtained by

$$
\int_{\Omega_{d}} \boldsymbol{\varepsilon}\left(\boldsymbol{v}_{j}\right)^{T} \boldsymbol{E} \boldsymbol{\varepsilon}\left(\boldsymbol{\phi}_{j}\right) d \Omega-\omega_{j}^{2} \int_{\Omega_{d}} \rho_{m} \boldsymbol{\phi}_{j}^{T} \boldsymbol{v}_{j} d \Omega=0 \text { for } \boldsymbol{\phi}_{j} \in V^{V i b}, \forall \boldsymbol{v}_{j} \in V^{V i b}
$$

where

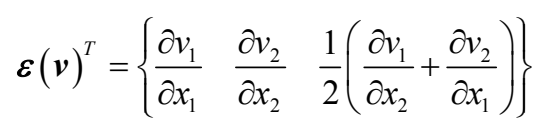

and

$$
V^{V i b}=\left\{\boldsymbol{v}=v_{i} \boldsymbol{e}_{i}: v_{i} \in H^{1}\left(\Omega_{d}\right) \text { with } \boldsymbol{v}=0 \text { on } \Gamma_{u}\right\}
$$

Note that the maximization of $f_{j}^{M, \text { In }}$, that is, the magnitude of eigen-mode $\phi_{j}$ at boundary $\Gamma_{t j}^{\mathrm{In}}$ is not always required. This maximization is taken into account to avoid the coincidence of boundary $\Gamma_{t j}^{\mathrm{In}}$, where the exciting traction $\boldsymbol{t}_{j}^{\mathrm{In}}$ is applied, with a node of the $j$-th eigen-mode $\phi_{j}$.

The third and final design requirement pertains to the global stiffness. Let us consider that the vibrating structure occupying the design domain $\Omega_{d}$ is subjected to the dynamic traction $\boldsymbol{t}_{j}^{\mathrm{In}}$ at boundary $\Gamma_{t j}^{\mathrm{In}}$ in order to vibrate the structure, and is also subjected to a reaction traction $\boldsymbol{t}_{j}^{\text {Out }}$ imposed by an object such as a workpiece at boundary $\Gamma_{t j}^{\text {Out }}$. In this case, two types of global stiffness are considered. One is the stiffness that maintains the structural shape when the dynamic traction $\boldsymbol{t}_{j}^{\mathrm{In}}$ is applied at boundary $\Gamma_{t j}^{\mathrm{In}}$ while boundary $\Gamma_{t j}^{\text {Out }}$ is fixed in the direction of $\boldsymbol{t}_{j}^{\text {Out }}$. Sufficient stiffness here is obtained by minimizing the absolute value of the dynamic mean compliance for the above boundary conditions ${ }^{(11)}$. However, this stiffness implementation is a subsidiary design specification for the optimum design, and does not require a rigorous evaluation of the mean compliance. Here, for the simplicity of optimization, this sufficient stiffness is 
implemented by minimizing the static mean compliance formulated in the following:

$$
\underset{r}{\operatorname{minimize}} l\left(\boldsymbol{u}_{j}^{\mathrm{In}}\right)=\int_{\Gamma_{t j}^{\mathrm{In}}} \boldsymbol{T}_{j}^{\mathrm{In}} \cdot \boldsymbol{u}_{j}^{\mathrm{In}} d \Gamma, \boldsymbol{u}_{j}^{\mathrm{In}} \in V_{j}^{\mathrm{In}} \text { for } j=1, \ldots, m_{s}
$$

where $\boldsymbol{T}_{j}^{\mathrm{In}}$ is the amplitude of $\boldsymbol{t}_{j}^{\mathrm{In}}$, and $\boldsymbol{u}_{j}^{\mathrm{In}}$ is the displacement field obtained by

$$
\begin{aligned}
& \int_{\Omega_{d}} \boldsymbol{\varepsilon}\left(\boldsymbol{v}_{j}^{\mathrm{In}}\right)^{T} \boldsymbol{E} \boldsymbol{\varepsilon}\left(\boldsymbol{u}_{j}^{\mathrm{In}}\right) d \Omega=\int_{\Gamma_{t j}^{\mathrm{In}}} \boldsymbol{T}_{j}^{\mathrm{In}} \cdot \boldsymbol{v}_{j}^{\mathrm{In}} d \Gamma \text { for } \boldsymbol{u}_{j}^{\mathrm{In}} \in V_{j}^{\mathrm{In}} \forall \boldsymbol{v}_{j}^{\mathrm{In}} \in V_{j}^{\mathrm{In}} \\
& V_{j}^{\mathrm{In}}=\left\{\boldsymbol{v}=v_{i} \boldsymbol{e}_{i}: v_{i} \in H^{1}\left(\Omega_{d}\right) \text { with } \boldsymbol{v}=0 \text { on } \Gamma_{u} \text { and }\left(\boldsymbol{T}_{j}^{\text {Out }}, \boldsymbol{v}\right)=0 \text { on } \Gamma_{t j}^{\text {Out }}\right\}
\end{aligned}
$$

and $\boldsymbol{T}_{j}^{\text {Out }}$ is the amplitude of $\boldsymbol{t}_{j}^{\text {Out }}$

The second type of stiffness is the stiffness that resists reaction traction $\boldsymbol{t}_{j}^{\text {Out }}$ at boundary $\Gamma_{t j}^{\text {Out }}$ while boundary $\Gamma_{t j}^{\mathrm{In}}$ is fixed in the direction of $\boldsymbol{t}_{j}^{\mathrm{In}}$. Sufficient stiffness is obtained by minimizing the static mean compliance under the above boundary conditions.

$$
\underset{r}{\operatorname{minimize}} l\left(\boldsymbol{u}_{j}^{\text {Out }}\right)=\int_{\Gamma_{t j}^{\text {Out }}} \boldsymbol{T}_{j}^{\text {Out }} \cdot \boldsymbol{u}_{j}^{\text {Out }} d \Gamma, \boldsymbol{u}_{j}^{\text {Out }} \in V_{j}^{\text {Out }} \text { for } j=1, \ldots, m_{s}
$$

where $\boldsymbol{u}_{j}^{\text {Out }}$ is the displacement field obtained by

$$
\begin{aligned}
& \int_{\Omega_{d}} \boldsymbol{\varepsilon}\left(\boldsymbol{v}_{j}^{\text {Out }}\right)^{T} \boldsymbol{E} \boldsymbol{\varepsilon}\left(\boldsymbol{u}_{j}^{\text {Out }}\right) d \Omega=\int_{\Gamma_{t j}^{\text {Out }}} \boldsymbol{T}_{j}^{\text {Out }} \cdot \boldsymbol{v}_{j}^{\text {Out }} d \Gamma \text { for } \boldsymbol{u}_{j}^{\text {Out }} \in V_{j}^{\text {Out }} \forall \boldsymbol{v}_{j}^{\text {Out }} \in V_{j}^{\text {Out }} \\
& V_{j}^{\text {Out }}=\left\{\boldsymbol{v}=v_{i} \boldsymbol{e}_{i}: v_{i} \in H^{1}\left(\Omega_{d}\right) \text { with } \boldsymbol{v}=0 \text { on } \Gamma_{u} \text { and }\left(\boldsymbol{T}_{j}^{\mathrm{In}}, \boldsymbol{v}\right)=0 \text { on } \Gamma_{t j}^{\text {In }}\right\}
\end{aligned}
$$

Since it is difficult to precisely evaluate the value of $\boldsymbol{T}_{j}^{\text {Out }}$, and this stiffness implementation is also a subsidiary design specification, we assume that $\boldsymbol{T}_{j}^{\text {Out }}$ is a unit vector in the engineering sense.

\subsection{Formulation of Optimization Problem}

As explained above, the four objective function formulated by Eqs. (9), (10), (14), and (17) must be taken into account in the design of such mechanical structures having dynamic functions. A multi-objective problem is formulated in order to obtain optimal solutions incorporating those objective functions.

As the first step, the following multi-objective function $f^{V}$ is formulated for Eq. (9).

$$
\underset{r}{\operatorname{minimize}} f^{V}=\sum_{j=1}^{m} w_{j}^{V} f_{j}^{V} 2
$$

where $w_{j}^{V}$ is the weighting coefficient for the $j$-th eigen-frequency matching. Next, the following multi-objective function $f^{M}$ is formulated for Eq. (10).

$$
\underset{r}{\operatorname{maximize}} f^{M}=\sum_{j=1}^{m_{s}}\left(w_{j}^{M, \text { In }} f_{j}^{M, \text { In 2 }}+w_{j}^{M, \text { Outmax }} f_{j}^{M, \text { Outmax 2 }}-w_{j}^{M, \text { Outmin }} f_{j}^{M, \text { Outmin2 }}\right)
$$

where $w_{j}^{M, \text { In }}, w_{j}^{M, \text { Outmax }}$, and $w_{j}^{M \text {,Outmin }}$ are the weighting coefficients for the maximization of the $j$-th eigen-mode in the direction of $\boldsymbol{t}_{j}^{\text {In }}$, the maximization of the $j$-th eigen-mode in the direction of $\boldsymbol{t}_{j}^{\text {Outmax }}$, and the minimization of the $j$-th eigen-mode in the direction of $\boldsymbol{t}_{j}^{\text {Outmin }}$, respectively. Finally, the following multi-objective function $f^{S}$ is formulated for Eqs. (14) and (17).

$$
\underset{r}{\operatorname{minimize}} f^{S}=\sum_{j=1}^{m_{s}} w_{j}^{S \text {, In }} l\left(\boldsymbol{u}_{j}^{\text {In }}\right)^{2}+w_{j}^{S, \text { Out }} l\left(\boldsymbol{u}_{j}^{\text {Out }}\right)^{2}
$$

where $w_{j}^{S \text {,In }}$ and $w_{j}^{S, \text { Out }}$ are the weighting coefficients for the $j$-th stiffness. Note that setting $w_{j}^{V}, w_{j}^{M, \text { In }}, w_{j}^{M, \text { Outmax }}, w_{j}^{M, \text { Outmin }}, w_{j}^{S \text {,In }}$, and $w_{j}^{S \text {,Out }}$ to 1 is sufficient to obtain reasonable optimal configurations as seen in the results of the many numerical tests we carried out. The formulation includes these weighting coefficients to ensure general applicability.

Furthermore, the above three objective functions are unified to a multi-objective function $f$, and the multi-objective optimization problem is formulated as follows:

$$
\underset{r}{\operatorname{minimize}} f=\left(1+w^{V} f^{V}\right) f^{S}-w^{M} f^{M}
$$

subject to 
Eqs. (3), (11), (15), and (18)

$$
g(r)=\int_{D}(1-r(x)) d \Omega-\Omega_{s} \leq 0
$$

where $w^{V}$ and $w^{M}$ are the weighting coefficients for $f^{V}$ and $f^{M}$, respectively, and $\Omega_{s}$ is the upper bound of the total volume constraint. Note that since the objective function $f^{S}$ must be taken into account to obtain reasonable optimal configurations that have physical meaning, a weighting coefficient for $f^{S}$ is not introduced. This is because if such a weighting coefficient were introduced, the stiffness effect might be negated if its value were set to zero. Thus, the multi-objective function in Eq. (23) is formulated in order to embed the effect of the stiffness regardless of the values of weighting coefficients $w^{V}$ and $w^{M}$. Taking a variation of the above multi-objective function yields

$$
\delta f=w^{V} f^{S} \delta f^{V}+\left(1+w^{V} f^{V}\right) \delta f^{S}-w^{M} \delta f^{M}
$$

This implies that the equivalent weighting coefficient for $\delta f^{V}$ is $w^{V} f^{S}$, and this weighting coefficient is large before convergence of the mean compliance, so that $f^{V}$ can achieve a sufficiently small value. That is, the convergence of the eigen-frequency specifications is advanced before the multi-objective function can achieve a local optimum. Once the eigen-frequency conditions largely satisfy the specifications, the objective function $f^{S}$ is improved since the equivalent weighting coefficient for $\delta f^{S}$ is $1+w^{V} f^{V}$, which is nearly equal to 1.0 after $f^{V}$ converges. (Further explication is available in Ref.(17).) Therefore, the multiplicative relation between $f^{V}$ and $f^{S}$ is considered in the above multi-objective formulation. Note that the results of numerical experiments showed no advantage to using a multiplicative formulation between $f^{M}$ and other objective functions, so an ordinary weighted-sum formulation is adopted. Also note that if a larger stiffness than that formulated by $f^{S}$ is required while the eigen-frequency objective function formulated by $f^{V}$ converges during the optimization process, the value of $w^{V}$ must be set smaller. If the eigen-mode objective function formulated by $f^{M}$ does not converge, the value of $w^{M}$ must be set larger.

\subsection{Optimization Algorithms}

Figure 2 shows a flowchart of the optimization. As shown in this figure, in the first step, the homogenized elasticity tensor and mass density $\boldsymbol{E}^{H}$ and $\rho_{m}^{H}$ are computed. In the second step, the eigen-value equation formulated as Eq. (11), and the two equilibrium equations formulated as Eqs. (15) and (18), are solved. In the third step, eigen-frequencies, eigen-mode shapes, the mean compliance, the objective function, and constraints are computed. In the fourth step, if the objective function converges, the optimization algorithm stops. Otherwise, the sensitivities of eigen-frequencies, eigen-mode shapes, mean compliance, and constraints with respect to the design variables are computed. In the fifth step, the design variables are updated using Sequential Linear Programming (SLP), and the procedure then returns the first step. 


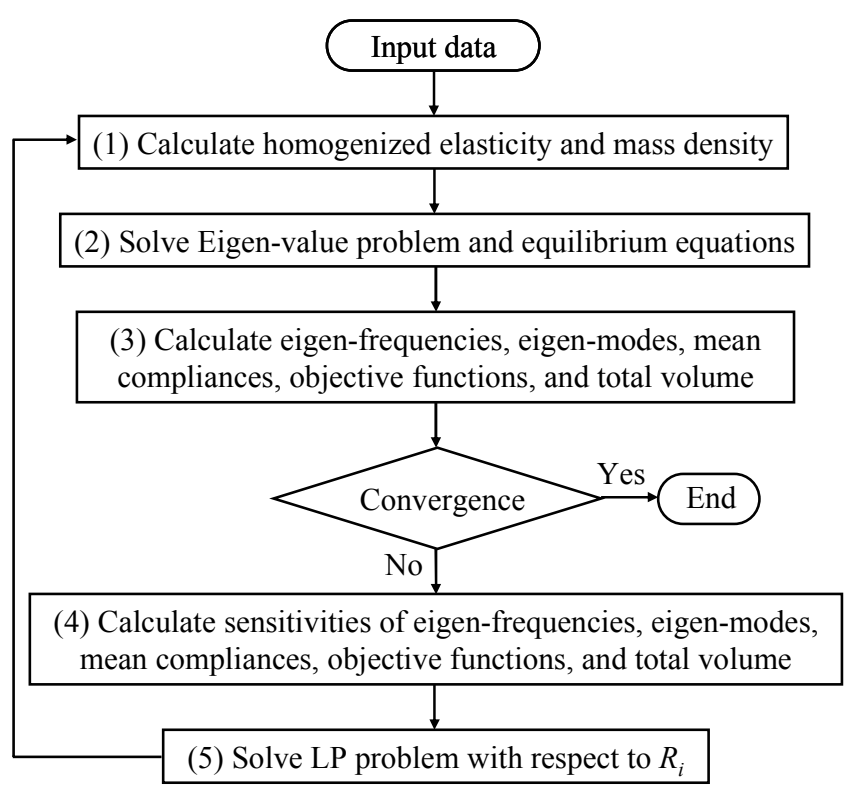

Fig. 2 Flowchart of optimization procedure

The sensitivity of the $j$-th eigen-value $\lambda_{j}$ where $\lambda_{j}=\omega_{j}{ }^{2}$ with respect to the $i$-th design variable $R_{i}^{(2)}$ is obtained by

$$
\frac{\partial \lambda_{j}}{\partial R_{i}}=\frac{\Phi_{j}^{T}\left(\frac{\partial \boldsymbol{K}}{\partial R_{i}}-\lambda_{j} \frac{\partial \boldsymbol{M}}{\partial R_{i}}\right) \Phi_{j}}{\Phi_{j}^{T} \boldsymbol{M} \Phi_{j}}
$$

where

$$
\begin{aligned}
& \Phi_{j}^{T} \frac{\partial \boldsymbol{K}}{\partial R_{i}} \Phi_{j}=\sum_{e=1}^{n_{i}} \int_{\Omega_{e}} \boldsymbol{\phi}_{j}^{T} \boldsymbol{B}^{T} \frac{d \boldsymbol{E}^{H}}{d r} \boldsymbol{B} \boldsymbol{\phi}_{j} N_{i}^{r}(\boldsymbol{x}) d \Omega \\
& \boldsymbol{\Phi}_{j}^{T} \frac{\partial \boldsymbol{M}}{\partial R_{i}} \boldsymbol{\Phi}_{j}=\sum_{e=1}^{n_{i}} \int_{\Omega_{e}} \boldsymbol{\phi}_{j}^{T} \boldsymbol{N}^{T} \frac{d \rho_{m}^{H}}{d r} \boldsymbol{N} \boldsymbol{\phi}_{j} N_{i}^{r}(\boldsymbol{x}) d \Omega
\end{aligned}
$$

and $\boldsymbol{K}$ and $\boldsymbol{M}$ are the stiffness matrix and mass matrix of the extended design domain $D$ after the finite element discretization is performed, $\Phi_{j}$ is the discretized $j$-th eigen-mode, $n_{i}$ denotes the number of elements associated with the $i$-th node, $N$ is a shape function matrix for the displacement interpolations, and $\boldsymbol{B}$ is a B-matrix such that $\boldsymbol{B}=\partial \boldsymbol{N}$.

The sensitivity of the $j$-th eigen-mode $\Phi_{j}$ with respect to the $i$-th design variable $R_{i}$ is calculated based on Wang's approach ${ }^{(18)}$. Let $\boldsymbol{f}$ be a dummy unit force vector computed as the discretized formulation of boundary traction formulated in Eq. (10), and $\boldsymbol{u}$ be a virtual displacement vector due to this boundary traction. The sensitivity of $\boldsymbol{f}^{T} \Phi_{j}$ in the discretized formulations is computed as

$$
\begin{aligned}
& \frac{\partial\left(\boldsymbol{f}^{T} \boldsymbol{\Phi}_{j}\right)}{\partial R_{i}}=\boldsymbol{u}^{T} \boldsymbol{K}\left(\boldsymbol{\Phi}_{j}^{s}+\sum_{k=1}^{m_{k}} d_{j k} \boldsymbol{\Phi}_{k}\right) \\
& =\boldsymbol{u}^{T}\left[\frac{\partial \lambda_{j}}{\partial R_{i}} \boldsymbol{M}-\frac{\partial \boldsymbol{K}}{\partial R_{i}}+\lambda_{j} \frac{\partial \boldsymbol{M}}{\partial R_{i}}\right] \boldsymbol{\Phi}_{j}+\boldsymbol{u}^{T} \boldsymbol{K} \sum_{k=1}^{m_{k}} d_{j k} \boldsymbol{\Phi}_{k}
\end{aligned}
$$

where $m_{k}$ is an appropriate number of eigen-modes associated with the lowest eigen-values, and $d_{j k}$ is computed as,

$$
d_{j k}=\lambda_{j} \frac{\Phi_{k}{ }^{T}\left(\frac{\partial \boldsymbol{K}}{\partial R_{i}}-\lambda_{j} \frac{\partial \boldsymbol{M}}{\partial R_{i}}\right) \Phi_{j}}{\lambda_{k}\left(\lambda_{j}-\lambda_{k}\right) \Phi_{k}{ }^{T} \boldsymbol{M} \Phi_{k}} \text { for } j \neq k
$$




$$
d_{i j}=-\frac{1}{2} \frac{\Phi_{j}^{T} \frac{\partial \boldsymbol{M}}{\partial R_{i}} \Phi_{j}}{\Phi_{j}^{T} \boldsymbol{M} \Phi_{j}}
$$

and $\boldsymbol{u}$ is obtained by

$$
\boldsymbol{u}=\boldsymbol{K}^{-1} \boldsymbol{f}
$$

The above formulation requires only one computation of the inverse matrix of $\boldsymbol{K}$, whereas the direct derivation of the sensitivities of $\Phi_{j}$ would require calculating the inverse matrix of $\boldsymbol{K}$ as many times as there are design variables, and the sensitivity analysis would likely then become intractable. Thus, the objective function formulated by Eq. (10) is advantageous for the optimization here. For the derivation of the sensitivities of the mean compliance, see the details in reference ${ }^{(17)}$.

In the step where design variables are updated using SLP during the optimization process, the relation between eigen-mode shapes before the update and those after the update must be monitored to determine whether or not the update operation has changed the sequence of eigen-frequencies and eigen-modes. Let $\boldsymbol{M}^{\{k\}}$ and $\Phi_{j}^{\{k\}}$ be the mass matrix and the $j$-th eigen-mode in the $k$-th update iteration, respectively. The $j$-th eigen-mode in the $(k+1)$-th update iteration, $\Phi_{j}^{\{k+1\}}$, is found using the following equation ${ }^{(19)}$ :

$$
\Phi_{j}^{\{k+1\} T} \boldsymbol{M}^{\{k+1\}} \Phi_{j}^{\{k\}} \approx 1
$$

Using the above equation, the relationship between the eigen-modes during the optimization and those of the initial configurations can be monitored, and the objective functions and sensitivities can be computed under a condition of appropriate eigen-frequency and eigen-mode ordering. For this reason, information concerning the initial design domain setting sequence is retained during optimization in the following examples.

\section{Numerical Examples}

Two numerical examples are presented in order to confirm the utility of the proposed method. In both these cases, the isotropic material has a Young's modulus = 200GPa, Poisson's Ratio $=0.3$, and a mass density $=7.8 \times 10^{3} \mathrm{Kg} / \mathrm{m}^{3}$. In the $\mathrm{FE}$ analysis, four node isoparametric full integration elements with bilinear shape functions are used. The magnitude of the amplitude of all applied forces is assumed to be a unit load. The weighting coefficients in the objective functions are set to 1.0 except for the specified values described in the following subsections. These specified values are searched for by numerical trial and error until optimal configurations that have physical meaning are obtained. In both examples, the initial configuration of the fixed design domain is set to a uniform microstructure distribution where $R_{i}(i=1, \ldots, n)$ is set to 0.9 .

\subsection{Relation between Eigen-frequency, Eigen-mode and Stiffness Settings, and Optimal Configurations}

Figure 3 shows the design domain, in which both the right and left sides of the fixed design domain $D$ are fixed. While several eigen-frequencies are required to conform to target values according to the eigen-frequency specification, the lowest eigen-mode along the direction $\boldsymbol{f}_{1}^{\text {In }}$ at point $P_{1}^{\text {In }}$ and along the direction $\boldsymbol{f}_{1}^{\text {Outmax }}$ at point $P_{1}^{\text {Outmax }}$ is to be maximized, and the lowest eigen-mode along the direction $f_{1}^{\text {Outmin }}$ at point $P_{1}^{\text {Outmin }}$ is to be minimized, according to the eigen-mode specifications shown in Fig. 3(a). Furthermore, the static stiffness along the direction of $\boldsymbol{f}_{1}^{\mathrm{In}}$ at point $P_{1}^{\mathrm{In}}$ is to be maximized while point $P_{1}^{\text {Out }}$ is fixed along the direction of $\boldsymbol{f}_{1}^{\text {Out }}$ to resist the applied force as shown in Fig. 3(b), and the static stiffness along the direction of $\boldsymbol{f}_{1}^{\text {Out }}$ at point $P_{1}^{\text {Out }}$ is to be maximized while point $P_{1}^{\text {In }}$ is fixed along the direction of $\boldsymbol{f}_{1}^{\text {In }}$ to resist the reaction force imposed by a workpiece, as shown in Fig. 3(c). The total volume constraint $\Omega_{\mathrm{s}}$ is set to $20 \%$ of the volume of the whole design domain. 


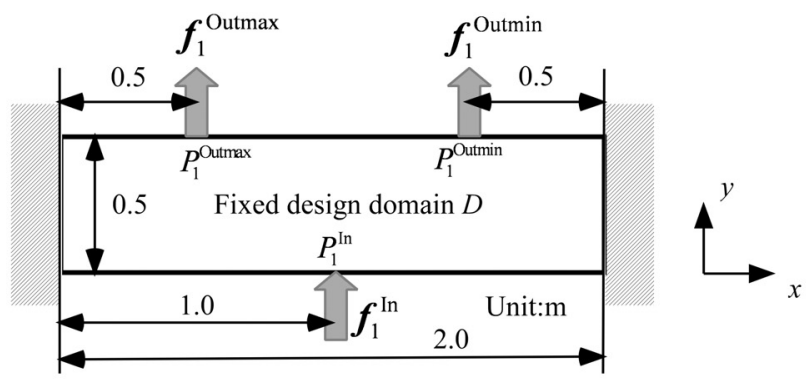

(a) Eigen-mode specifications

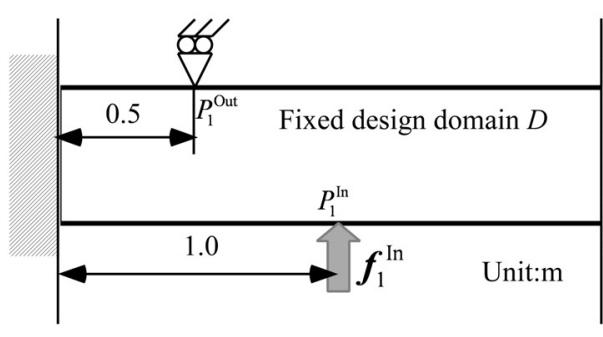

(b) Stiffness specification for input force

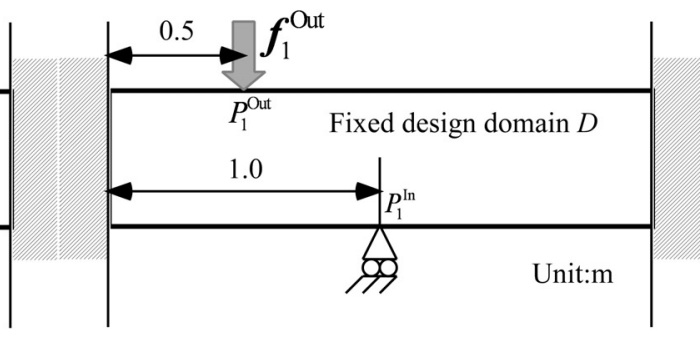

(c) Stiffness specification for reaction force

Fig. 3 Design domain for simple model 1

Figure 4 shows the optimal configurations. The shading of black and white in the figures describes the distribution of the normalized density, where white represents a void, i.e., $r=1$, and black a solid, $r=0$, shown in Fig. 1 . The same shading method is also used in subsequent figures. Table 1 lists the values from the lowest (first) to the fourth eigen-frequency of the optimal configurations in Fig. 4.

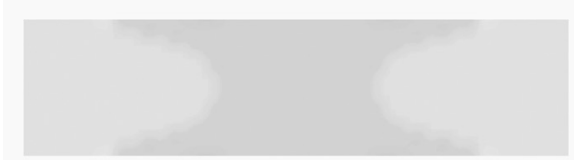

(a) Lowest target eigen-frequency $=200 \mathrm{~Hz}$, eigen-mode and stiffness specifications not considered

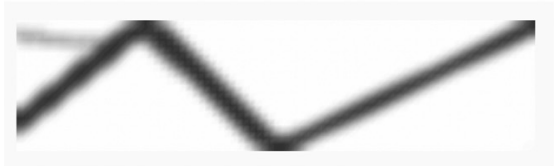

(b) Lowest target eigen-frequency $=200 \mathrm{~Hz}$, stiffness specification considered, eigen-mode specification not considered

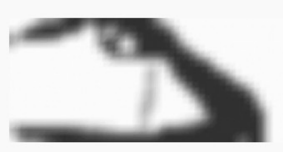

(c) Lowest target eigen-frequency $=50 \mathrm{~Hz}$, stiffness specification considered, eigen-moede specification not considered

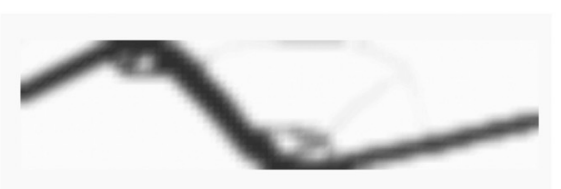

(d) Lowest target eigen-frequency $=100 \mathrm{~Hz}$, stiffness specification considered, eigen-moede specification not considered

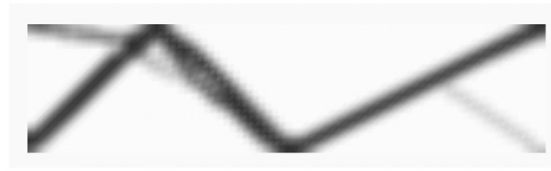

(e) Lowest target eigen-frequency $=300 \mathrm{~Hz}$, stiffness specification considered, eigen-moede specification not considered

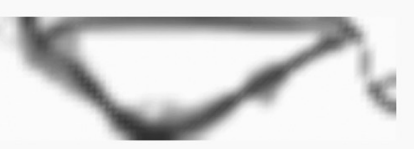

(f) Lowest, 2nd, and 3rd target eigen-frequenies = $50 \mathrm{~Hz}, 100 \mathrm{~Hz}$, and $200 \mathrm{~Hz}$, stiffness specification considered, eigen-moede specification not consid

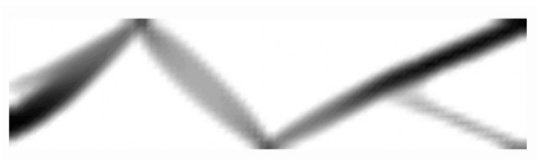

(g) Lowest target eigen-frequency $=200 \mathrm{~Hz}$, eigen-mode and stiffness specifications considered



Fig. 4 Optimal configurations of simple model 1 
Table 1 Eigen-frequencies of optimal configurations shown in Fig. 4

\begin{tabular}{c|cccc} 
& \multicolumn{4}{|c}{ Eigen-frequencies (Hz) } \\
Figs. & 1st & 2nd & 3rd & 4th \\
\hline (a) & 200.39 & 467.82 & 544.79 & 772.90 \\
(b) & 198.89 & 261.46 & 513.50 & 581.90 \\
(c) & 51.02 & 194.24 & 380.81 & 423.58 \\
(d) & 100.18 & 315.64 & 472.94 & 536.37 \\
(e) & 297.01 & 347.08 & 522.31 & 584.59 \\
(f) & 50.65 & 117.59 & 211.66 & 477.95 \\
(g) & 200.52 & 414.83 & 504.23 & 646.50
\end{tabular}

In Fig. 4(a), only the lowest target eigen-frequency is set to $200 \mathrm{~Hz}(m=1)$ while the eigen-mode and stiffness specifications shown in Fig. 3(a), (b), and (c) are not considered. In Fig. 4(b), (c), (d), and (e), only the lowest target eigen-frequency is set to $200 \mathrm{~Hz}, 50 \mathrm{~Hz}, 100 \mathrm{~Hz}$, and $300 \mathrm{~Hz}$, respectively, and the stiffness specifications shown in Fig. 3(b) and (c) are considered while the eigen-mode specifications shown in Fig. 3(a) are not considered. In all these cases, the weighting coefficient $w^{V}$ is set to 10.0.

In Fig. 4(f), the lowest, second, and third target frequencies are set to $50 \mathrm{~Hz}$, $100 \mathrm{~Hz}$, and $200 \mathrm{~Hz}$, respectively $(m=3)$, and the stiffness specifications shown in Fig. 3(b) and (c) are considered while the eigen-mode specifications shown in Fig. 3(a) are not considered. In this case, the weighting coefficient $w^{V}$ is set to 20.0.

In Fig. 4(g), only the lowest target eigen-frequency is set to $200 \mathrm{~Hz}(m=1)$, and the eigen-mode and stiffness specifications shown in Fig. 3(a), (b), and (c) are considered. In this case the weighting coefficients $w^{V}$ and $w^{M}$ are set to 10.0 and 0.1 , respectively. Fig. 4 (h) shows the lowest eigen-mode shape of this optimal configuration.

As shown in Table 1, no sequence changes from the lowest (first) eigen-frequency to the fourth occur. It is noteworthy that even though only the lowest eigen-frequency is specified in Fig. 4(b) (e), reordering does not occur, and the design specifications are satisfied.

Comparing Fig. 4(a) with Fig. 4(b), the optimal configuration where the stiffness specifications are not considered, Fig. 4(a) reveals significant gray scale and is not appropriate in the engineering sense, but the optimal configuration where the stiffness specifications are considered, namely, Fig. 4(b), is clear, although both results satisfy the eigen-frequency specification according to Table 1.

As shown in Fig. 4(b) (e) and Table 1, the lowest-eigen frequencies of the optimal configurations reaches their target values in all cases from $50 \mathrm{~Hz} \sim 300 \mathrm{~Hz}$, and all optimal configurations are clear, with no checkerboard patterns observed. The optimal configuration in Fig. 4(f) is also clear, and is obtained as a solution that has physical meaning. Furthermore, the lowest, second, and third frequencies of this configuration closely approach their respective target frequency, as specified. The optimal configuration in Fig. 4(g) is also clear, and satisfies the eigen-frequency and eigen-mode specifications illustrated in Fig. 4(h) and detailed in Table 1.

Thus it is confirmed that the proposed method can provide optimal configurations for mechanical structures that simultaneously satisfy eigen-frequency, eigen-mode, and stiffness specifications.

\subsection{Design of Vibromotor Using Second Eigen-mode}

Figure 5 shows the design domain for a simple vibromotor. As shown in this figure, the second eigen-mode along the direction $\boldsymbol{f}_{2}^{\text {Outmax }}$ at point $P_{2}^{\text {Outmax }}$, along the direction $\boldsymbol{f}_{2,1}^{\mathrm{In}}$ at point $P_{2,1}^{\mathrm{In}}$, and along the direction $\boldsymbol{f}_{2,2}^{\mathrm{In}}$ at point $P_{2,2}^{\mathrm{In}}$ is to be 
maximized, and the second eigen-mode along the direction $\boldsymbol{f}_{2}^{\text {Outmin }}$ at point $P_{2}^{\text {Outmin }}$, which is same as $P_{2}^{\text {Outmax }}$ in the problem, is to be minimized according to the eigen-mode specification shown in Fig. 5(a) in order to move a workpiece in the horizontal direction using a resonance phenomenon, while the lowest and second eigen-frequencies are required to conform to target values, $350 \mathrm{~Hz}$, and $500 \mathrm{~Hz}$, respectively, according to the eigen-frequency specification. Further, the static stiffness along the direction of $\boldsymbol{f}_{2,1}^{\text {In }}$ at point $P_{2,1}^{\mathrm{In}}$ and along the direction $\boldsymbol{f}_{2,2}^{\mathrm{In}}$ at point $P_{2,2}^{\mathrm{In}}$ is to be maximized to resist the applied force as shown in Fig. 5(b), and the static stiffness along the direction of $\boldsymbol{f}_{2}^{\text {Out }}$ at point $P_{2}^{\text {Out }}$ is to be maximized while point $P_{2,1}^{\mathrm{In}}$ is fixed along the direction of $\boldsymbol{f}_{2,1}^{\mathrm{In}}$, and point $P_{2,2}^{\mathrm{In}}$ is fixed along the direction of $\boldsymbol{f}_{2,2}^{\mathrm{In}}$ to resist the reaction force imposed by the workpiece, as shown in Fig. 5(c). Note that no fixed condition representing the workpiece reaction force is imposed in Fig. 5(b), and the vertical force $\boldsymbol{f}_{2}^{\text {Out }}$ is assumed in the engineering sense since workpiece reaction forces resist precise evaluation in these design settings. The total volume constraint $\Omega s$ is set to $20 \%$ of the volume of the whole design domain. The weighting coefficients $w^{V}$ and $w^{M}$ are set to 5.0 and 0.1 , respectively.

Figure 6(a) shows the optimal configurations, and Fig. 6(b) shows the eigen-mode shape corresponding to the second eigen-frequency. The values of the eigen-frequencies of the optimal configurations, from the lowest (first) to the fourth, are $355.32 \mathrm{~Hz}, 503.34 \mathrm{~Hz}, 524.60 \mathrm{~Hz}$, and $739.51 \mathrm{~Hz}$. As shown in this figure, the optimal configuration is also clear, and free from checkerboard patterns. Further, we see that the result satisfies the eigen-frequency and eigen-mode specifications. Indeed, the results of numerical experiments here show that in these design settings, the second mode shape is more useful than the first mode shape for obtaining the desired mode in the optimal configuration. Thus it is confirmed that the use of a higher order eigen-mode may be of benefit for the design of mechanical structures having dynamic functions, such as vibromotors.

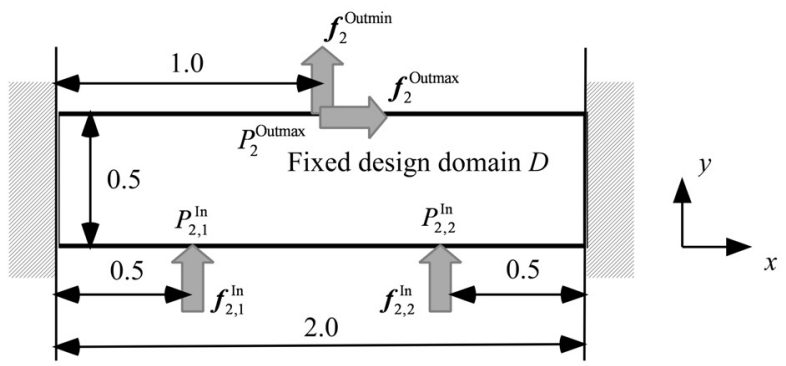

(a) Eigen-mode specifications

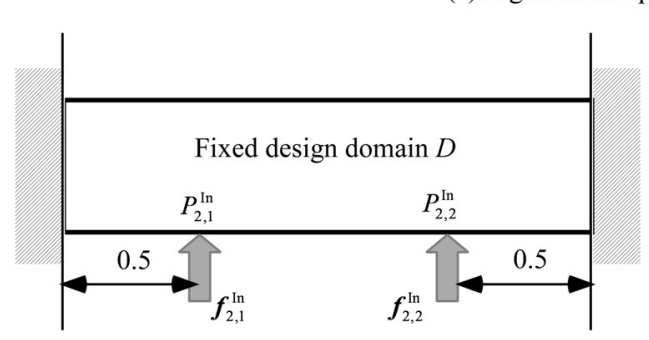

(b) Stiffness specification for input force

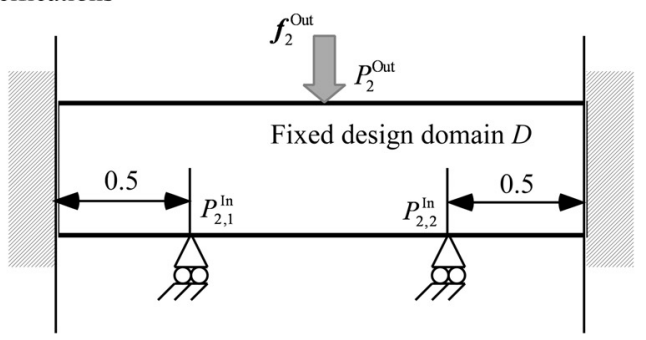

(c) Stiffness specification for reaction force

Fig. 5 Design domain for simple model 2 


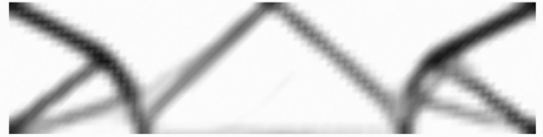

(a) Lowest and 2nd arget eigen-frequencies = $350 \mathrm{~Hz}$ and $500 \mathrm{~Hz}$, eigen-mode and stiffness specifications considered

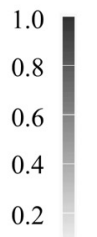

0.0

Normalized density

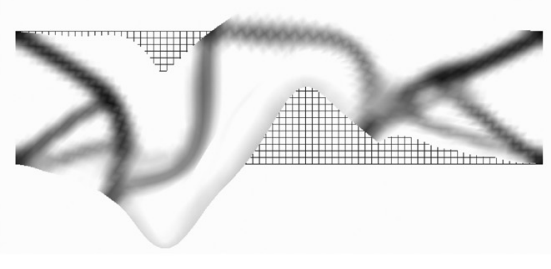

(b) 2nd eigen-mode shape of optimal configuration

Fig. 6 Optimal configurations of simple model 2

\section{Conclusions}

In this research, we developed a new structural topology optimization method that can implement desired eigen-frequencies and their corresponding eigen-modes for the design of mechanical structures having new dynamic functions. We achieved the following:

(1) A new topology optimization method based on the relaxation of the design domain where continuous material distributions are assumed using a continuous interpolation function was extended to dynamic problems.

(2) The design requirements and specifications concerning eigen-frequencies, eigen-mode shapes and stiffnesses were clarified so that additional desired dynamic functions could be implemented in the mechanical structures, and corresponding objective functions to satisfy these design specifications were formulated. A new multi-objective function was formulated for use in finding optimal configurations that incorporate all objective functions.

(3) A topology optimization algorithm was constructed based on the new relaxation scheme and optimization formulations.

(4) Two simple numerical examples were provided in order to examine the characteristics of the resulting optimal configurations. It was confirmed that the proposed method can produce mechanical structures that have the eigen-frequencies and eigen-modes specified in the design.

The authors sincerely appreciate the support received from the 21st century COE program "Center of Excellence for Research and Education on Complex Functional Mechanical Systems". The authors are also extremely grateful for the support received in Korea Research Foundation Grant (KRF-2003-042-D00009).

\section{References}

(1) Diaz, A.R. and Kikuchi, N., Solutions to shape and topology eigenvalue optimization using a homogenization method, Int. J. Numer. Meth. Engrg., Vol. 35(1992), pp. 1487-1502.

(2) Ma, Z.-D., Kikuchi, N., Cheng, H.-C., T, Topological design for vibrating structures, Comput. Methods Appl. Mech. Engrg., Vol .121(1995), pp. 259-280.

(3) Pedersen, N. L., Maximization of eigenvalues using topology optimization, Struct. Multi. Optim.,Vol. 20(2000), pp. 2-11.

(4) Allarie, G., Aubry, S., and Jouve, F., Eigenfrequency optimization in optimal design, Comput. Methods Appl. Mech. Engrg ., Vol. 190(2001), pp. 3565-3579.

(5) Howe, R.T. and Boser, B. E., Surface micromachined accelerometers, IEEE J. Solid-State Circuit, Vol. 31(1996), pp. 366-375.

(6) Jose, K. A., Suh, W. D., Xavier, P. B., Varadan, V. K., and Varadan, V.V., Surface acoustic wave MEMS gyroscope, Wave Motion, Vol. 36(2002), pp. 367-381.

(7) Bannon, F. D. III, Clark, J. R., and Nguyen, C.T.-C., HF microelectromechanical filters, J. Microelectromechanical Systems, Vol.35(2000), pp. 512-526.

(8) Saitou, K., Wang, D.-A., and Wou, S. J., J., Externally resonated linear microvibromotor for microassmbly, Microelectromechanical Systems, Vol .9(2000), pp. 336-346. 
(9) Lee, A. P. and Pisano, A. P.,J., Polysilicon angular microvibromotors, Microelectromechanical Systems, Vol.1(1992), 621-629.

(10) Bendsøe, M.P. and Kikuchi, N., Generating optimal topologies in structural design using a homogenization method, Comput. Methods Appl. Mech. Engrg ., Vol. 71(1988), pp. 197-224.

(11) Nishiwaki, S., Saitou, K., Min, S., and Kikuchi, N., Topological design considering flexibility under periodic loads, Struct. Optim., Vol. 19(2000), pp. 4-16.

(12) Tcherniak, D., Topology optimization of resonating structures using SIMP method, Int. J. Numer. Meth. Engrg., Vol. 54( 2002), pp. 1605-1622.

(13) Suzuki, K. and Kikuchi, N., A homogenization method for shape and topology optimization, Comput. Methods Appl. Mech. Engrg .,Vol. 93(1991), pp. 291-318.

(14) Sigmund, O. and Petersson, . Numerical instabilities in topology optimization: A survey on procedures dealing with checkerboards, mesh-dependencies and local minima, J., Struct. Optim., Vol. 16(1998), pp. 68-75.

(15) Bourdin, B., Filters in topology optimization, Int. J. Numer. Meth. Engrg., Vol. 50(2001), pp. 2143-2158.

(16) Fujii, D. and Kikuchi, N., Improvement of numerical instabilities in topology optimization using SLP method, Struct. Optim., Vol. 19(2000), pp. 113-121.

(17) Nishiwaki, S., Min, S., Yoo, J., and Kikuchi, N., Optimal structural design considering flexibility, Comput. Methods Appl. Mech. Engrg., Vol. 190 (2001), pp. 4457-4504.

(18) Wang, B. P., Improved approximate methods for computing eigenvector derivative in structural dynamics, AIAA Journal, Vol. 29(1991), pp. 1018-1020.

(19) Allemang, R. J., The modal assurance criterion - Twenty years of use and abuse, Sound and Vibration, Vol. 37(2003), pp. 14-23. 